Universidad de Lima

Facultad de Psicología

Carrera de Psicología

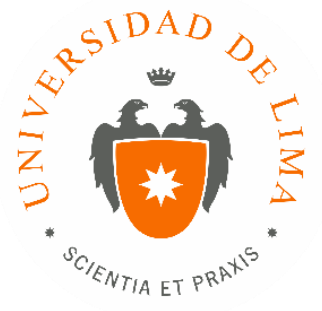

\title{
ESTRATEGIAS DE SELECCIÓN Y \\ CAPACITACIÓN PARA LA GESTIÓN DEL TALENTO
}

Trabajo de suficiencia profesional para optar el título profesional de Licenciado en Psicología

Melissa Grace Chávez Simeón

Código 20072233

Lima - Perú

Febrero de 2019 


\section{ESTRATEGIAS DE SELECCIÓN Y CAPACITACIÓN PARA LA GESTIÓN DEL TALENTO}




\section{TABLA DE CONTENIDO}

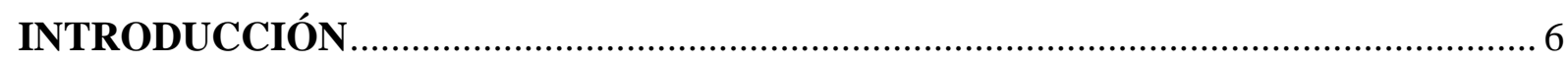

CAPÍTULO I: IDENTIFICACIÓN DEL PROBLEMA ............................................. 7

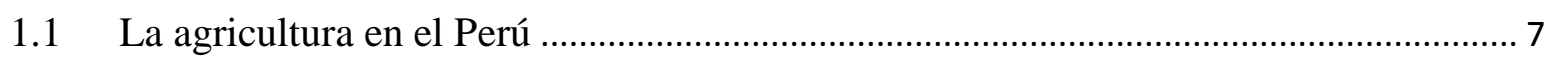

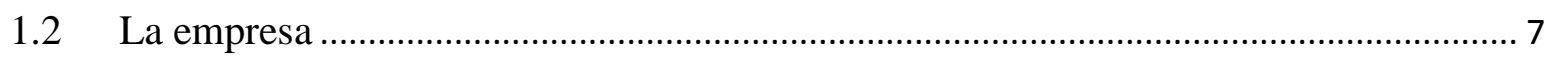

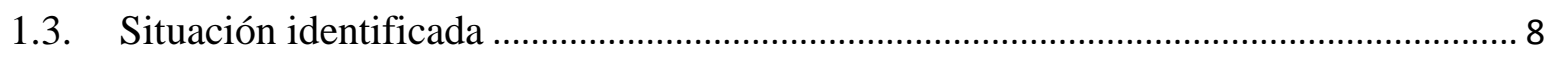

CAPÍTULO II: DESCRIPCIÓN DE LAS ACTIVIDADES REALIZADAS .................. 10

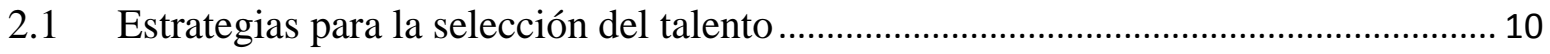

2.2 Estrategias para orientar la formación de los colaboradores ........................................ 12

CAPÍTULO III: RESULTADOS DE LA INTERVENCIÓN ........................................ 15

3.1 Resultado de las estrategias implementadas para la selección del talento........................ 15

3.2 Resultado de las estrategias para orientar la formación de los colaboradores ................. 16

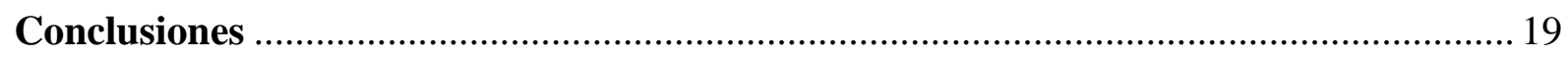

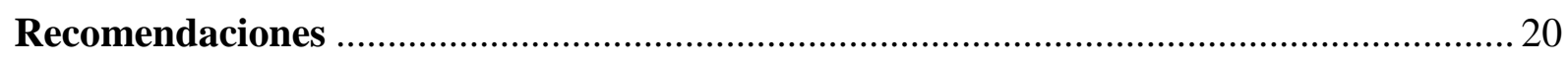

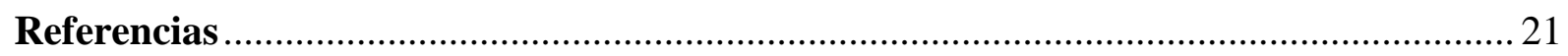




\section{ÍNDICE DE APÉNDICES}

Apéndice 1: Comparación entre Régimen Laboral General y Régimen Laboral Agrario ...... 24 


\section{INTRODUCCIÓN}

La agricultura se ha convertido en uno de los actores principales para el desarrollo económico a nivel mundial, siendo una de sus características la necesidad de mano de obra (Food and Agriculture Organization, 2000). En el Perú, la necesidad de contratación de personas para atender las necesidades de este sector, se incrementó durante los últimos años (Banco Mundial, 2017). Esta situación motiva a los responsables de la gestión del talento humano en las empresas a implementar medidas diferentes a las que normalmente podrían considerarse en un área de Recursos Humanos de la capital.

El presente documento fue elaborado con la autorización de la Gerencia General de la empresa y expone las estrategias implementadas para una gestión del talento adaptadas a las características y necesidades de la organización, a partir de los procesos de selección y capacitación. Para lograrlo, fue importante entender la operación, adaptarse a las condiciones del ambiente, ganar la confianza de los colaboradores y trabajar de la mano con las jefaturas y gerencias de las diferentes áreas, proponiendo e implementando metodologías nuevas con la confianza de que se alcanzarían los resultados esperados. Otro factor importante fue el compromiso de la empresa con el bienestar y el desarrollo de sus trabajadores.

El primer capítulo expone la situación encontrada al inicio de la gestión. El segundo capítulo presenta las actividades realizadas como estrategias para alcanzar mejores resultados. En el tercer capítulo se detallan los resultados obtenidos con la implementación de estas estrategias, tanto cuantitativos como cualitativos. Finalmente, se presentan las conclusiones y recomendaciones a partir de lo antes expuesto. 


\section{CAPÍTULO I: IDENTIFICACIÓN DEL PROBLEMA}

Según la FAO (2000), el sector agrícola es una actividad de gran importancia a nivel mundial, por ser un impulsor de la economía de los países en desarrollo y generador de oportunidades de empleo a una población significativa.

\subsection{La agricultura en el Perú}

En el Perú, este sector ha tenido un notable y sostenido crecimiento durante los últimos años. El Producto Bruto Interno (PBI) agrícola creció un promedio de 3.3 por ciento del año 2000 al 2015 (Ministerio de Agricultura y Riego, 2018) y la cantidad de personas empleadas creció cerca de medio millón entre los años 1994 y 2012 (Banco Mundial, 2017). En este sector, coexisten unidades productivas de diversos tamaños, que utilizan mano de obra familiar y/o contratada (Banco Mundial, 2017), así como empresas informales y formales. Estas últimas se rigen a las condiciones del régimen laboral agrario (Ley 27360). Las características de este régimen se pueden observar en el Apéndice $\mathrm{N}^{\circ} 1$.

\subsection{La empresa}

La empresa se fundó en el año 1994 como una empresa familiar con el objetivo de producir fruta de exportación. En sus campos ubicados en el Valle del río Huaura se cultivan palta y cítricos. La calidad de sus productos es respaldada por certificaciones internacionales y el proceso de exportación de estos productos es asumido por un consorcio de productores a nivel nacional, a la que se encuentra asociada. Para atender su operación, requiere 150 personas de manera permanente y más de 350 durante los meses de cosecha. El $80 \%$ es Operario contratado según el 
régimen laboral agrario, 55\% mujeres y $45 \%$ de hombres, desde personas no alfabetizadas hasta con estudios superiores inconclusos, en rango de edades de 18 a 58 años, migrantes de provincias, con y sin discapacidad. El 95\% proviene de centros poblados circundantes.

Se orienta al bienestar del personal y apuesta por el desarrollo de sus trabajadores, posee una infraestructura cómoda y moderna para uso de sus trabajadores, con baños en campo, un comedor con televisión satelital, espacios deportivos y equipos de entretenimiento. En el año 2014 inició un modelo de gestión empresarial; contrataba proveedores para selección de personal, medición de clima organizacional y capacitación en habilidades blandas, hasta que en el año 2016 creó el puesto de Coordinadora de Selección y Desarrollo asignado a la autora del reporte. Esta área asumió las responsabilidades de selección, capacitación y desarrollo en la organización, reportando a la Gerencia General.

\subsection{Situación Identificada}

Se contaba con un manual de funciones del área administrativa y se encontraba en proyecto la elaboración a nivel organizacional. El reclutamiento se realizaba por referencias de trabajadores y publicaciones radiales, las evaluaciones se desarrollaban en locales comunales de los centros poblados. La oferta de los "contratistas", intermediarios entre el "trabajador" y la unidad que requería de mano de obra, quienes aparentaban un pago superior y sin beneficios legales, así como el desconocimiento de los beneficios ofrecidos por la empresa eran aspectos influyentes en los posibles postulantes. El resultado se reflejaba en la dificultad para atraer mano de obra en temporada de cosecha, momento clave para la empresa, poniendo en riesgo la producción del año. 
El personal Operario contratado iniciaba sus labores de inmediato, se asumía que contaba con experiencia y que sus errores serían corregidos por los Supervisores de Campo; la ausencia de una inducción provocaba una adaptación lenta al puesto y errores de cosecha que impactaban en la calidad del producto. Las capacitaciones relacionadas al proceso productivo estaban a cargo del área de Producción, la capacitación de Operarios se realizaba con una metodología tradicional y orientada a cumplir los temas, las evidencias se registraban en formatos físicos y había dificultad para reunir el historial de un trabajador. Las capacitaciones para otros puestos eran solicitadas por el trabajador o sugeridas por sus jefaturas y en algunos casos autorizadas por el Directorio. La ausencia de un programa de capacitaciones impedía planificar y controlar la mejora de las capacidades en los trabajadores, la metodología no garantizaba el aprendizaje y el procedimiento de autorización generaba conflicto, impactando en la producción y en el clima organizacional.

Los puestos de supervisión y jefatura relacionados al campo fueron ocupados progresivamente por trabajadores con mayor tiempo de permanencia en la empresa y de confianza de los superiores por considerarlos buenos trabajadores. El resultado de la medición de clima organizacional del año 2015 mostraba un 50\% de aprobación al concepto de trato igualitario de los jefes y en conversación con los Operarios expresaban la existencia de favoritismo y asignación desigual de tareas. La carencia de una preparación previa para que estos nuevos supervisores o jefes asuman el nuevo cargo los expuso a situaciones negativas durante su gestión.

Por lo antes expuesto, el problema principal era la ausencia de procesos de selección y capacitación que permitan gestionar el talento de forma efectiva, entendiendo las necesidades del negocio y comprometiéndose con los objetivos planteados por la organización. 


\section{CAPÍTULO II: DESCRIPCIÓN DE LAS ACTIVIDADES Y TAREAS REALIZADAS}

A partir del problema identificado, se dio mayor importancia a los procesos del área de Selección y Desarrollo que tenían mayor impacto en la Producción, el centro del negocio. Se realizaron actividades relacionadas a la selección de los mejores candidatos para ocupar los puestos de Operario, a la mejora de sus competencias y al refuerzo de las capacidades de liderazgo en sus jefes directos. El objetivo fue implementar estrategias de selección y capacitación que contribuyan con una gestión más efectiva del talento.

\subsection{Estrategias para la selección del talento}

Enmarcado en la función de búsqueda y selección de personal, su objetivo fue la atracción de personas con las competencias para cumplir la función más importante del proceso productivo: la cosecha. Para lograrlo se realizaron diversas actividades. Conocer la operación y sus necesidades. Antes de iniciar con la gestión, se realizaron visitas a campo para interactuar con el personal y, por medio de la técnica de observación, conocer el proceso. Para Dessler y Varela (2011), la observación directa es útil cuando los puestos consisten en actividades físicas observables. Posteriormente, en reunión con la jefatura y gerencia del área, se definieron las funciones del puesto, el resultado esperado para el puesto y las características de la persona que lo ocuparía. Este paso tuvo como objetivo conocer la operación y definir el perfil de puesto requerido.

Mejorar la técnica de reclutamiento. Para Chiavenato (2017), las técnicas de reclutamiento son métodos para comunicar la existencia de una oportunidad de trabajo, entre ellas la recomendación de candidatos por trabajadores de la empresa y 
los anuncios en periódicos y revistas. Con el objetivo de atraer la mayor cantidad de postulantes, se elaboraron anuncios con lenguaje sencillo y énfasis en los beneficios de la empresa y de ley, dando facilidades de transporte y dos turnos de evaluación. Se comunicaron por dos emisoras radiales en horarios de mayor audiencia (de 3 a 7 de la mañana y de 5 a 9 de la noche), se publicaron en los centros poblados y se informó verbalmente a los trabajadores, motivándolos a invitar a su comunidad.

Mejorar el proceso de evaluación. Para Cuestas (2017), la imagen de la empresa es una ventaja o desventaja para atraer y conservar empleados. En esta situación, se quiso considerar la imagen de la empresa como una ventaja. El proceso iniciaba con la presentación de la empresa (historia, logros, objetivos, beneficios para los trabajadores), las condiciones de contratación y el proceso de selección. Las evaluaciones incluían la aplicación del Test de la Persona bajo la lluvia (Querol y Alcañiz, 2005) y simulaciones basadas en la metodología del Centro de Evaluación (Assessment Center); se sumaban los resultados del proveedor de verificación de referencias personales. Como lo menciona Vargas (2018), el Assessment Center y las evaluaciones psicológicas son métodos eficientes que pueden complementarse. En este sentido, el objetivo fue incrementar en los postulantes su deseo de pertenecer a la empresa y reunir mayor información que permita decidir su contratación.

Registrar la información y controlar el proceso. Se elaboró un archivo digital con información de los candidatos (nombres y apellidos, DNI, fecha de nacimiento, edad, género, teléfono de contacto) y el resultado en cada etapa del proceso. Se tuvo como objetivo contar con una base de candidatos útil para los procesos futuros, pues como lo señala Chiavenato (2017), la Consulta de archivo de candidatos es una técnica de reclutamiento donde se registran los datos de aquellos que no fueron seleccionados, 
involucra un bajo costo y requiere poco tiempo en su uso. Además, esta base de datos podía proporcionar información sobre la efectividad de los procesos de selección.

\subsection{Estrategias para orientar la formación de los colaboradores}

Como parte de la función de capacitación, este proceso tuvo como objetivo facilitar a los colaboradores conocimientos y desarrollar en ellos habilidades para que puedan realizar su trabajo de la mejor manera. Dessler y Varela (2011) consideran que la capacitación es un conjunto de métodos para dar a los trabajadores, nuevos o no, las habilidades que necesiten para realizar sus labores. Por ello se realizaron las actividades detalladas en los párrafos siguientes.

Identificar las necesidades reales de capacitación. Esta etapa tuvo como objetivo contar con información clara y concreta para orientar la formación de los colaboradores. Se identificaron factores de origen externo e interno; externos, como requeridos en normativas vigentes como certificaciones, legislación laboral, seguridad, salud y medio ambiente; internos, como resultados de rendimiento de cosecha, reportes de calidad y resultado de medición de clima organizacional. Posteriormente, se observaron las tareas realizadas en campo y se desarrollaron reuniones con las jefaturas y gerencias de áreas para conocer sus necesidades y establecer los objetivos. Siguiendo a Chiavenato (2017), la observación, la solicitud de supervisores y gerentes y los informes periódicos, forman parte de los once medios principales para detectar las necesidades de capacitación.

Programar la formación en un periodo anual. Se tuvo como objetivo elaborar un plan de capacitación a partir de las necesidades identificadas, para luego realizar el seguimiento a su implementación. Se elaboró una base de datos con nombre de la gerencia, nombre del participante, tema, necesidad identificada, objetivo de la 
capacitación, método, capacitador sugerido, tiempo y costo estimado; este programa debía ser aprobado por las jefaturas y gerencias de áreas antes de su implementación, pudiendo tener modificaciones durante el periodo anual, en función a los requerimientos de las áreas. Robbins y Judge (2017) consideran que los programas de capacitación y desarrollo orientan al logro de nuevos niveles de habilidades y agregan valor a la organización.

Capacitar al personal ingresante. Para Dessler y Varela (2011), la inducción otorga a los nuevos trabajadores información básica, necesaria para realizar sus labores de forma satisfactoria. En este sentido, se consideró la inducción con dos componentes; inducción a la empresa, a cargo del área de Personal y orientado a todos los trabajadores; y al puesto, dictada por los Jefes de Cultivo y orientada a los Operarios. Entonces, antes de iniciar sus labores, todo Operario contratado era capacitado en temas relacionados a higiene general y en campo, procedimiento de cosecha, uso seguro de herramientas de cosecha y uso seguro de productos químicos y equipos de protección personal. El objetivo era acelerar la adaptación de la persona a la empresa y al puesto y reducir errores durante la cosecha.

Mejorar la metodología de capacitaciones internas a Operarios. Se tuvo como objetivo orientar la metodología de capacitación al logro del aprendizaje. Para Imbernón (2016), la intencionalidad pedagógica y su potencial para diseñar e implementar programas que permitan el aprendizaje, son características importantes de la formación. Los temas de inducción al puesto fueron replicados a todo el personal Operario, las capacitaciones se desarrollarían en campo, con grupos reducidos y con materiales relacionados al tema. Se procuró que los capacitadores comprendan la importancia de esta metodología y se les facilitó una estructura que les ayude con el dictado del tema; la estructura consistía en dinámica de inicio, 
preguntas sueltas a los participantes para identificar su conocimiento, explicación de forma organizada y con un lenguaje sencillo apoyado en los materiales y un debate sobre el impacto del tema en la organización. Pimienta (2012) considera que el indagar sobre los conocimientos previos, promover la comprensión mediante la organización de la información y realizar debates grupales son estrategias capaces de enriquecer la formación.

Mejorar las capacidades de liderazgo de los Supervisores de Campo. A partir de los resultados de medición de clima organizacional en el año 2017, se establecieron acciones con el Jefe de Producción y Gerencia Agrícola para mejorar la gestión de los Supervisores de Campo. El objetivo fue capacitar a los Supervisores de Campo en temas de habilidades blandas, como herramientas para su gestión. En el mes de enero del año 2018, participaron del taller de liderazgo, programado inicialmente para jefes y gerentes. En febrero del mismo año participaron de cuatro talleres en solución de problemas, manejo de conflictos, comunicación efectiva y trabajo en equipo. Para medir el impacto de este trabajo, en los meses de mayo y julio, se desarrollaron encuestas a los operarios respecto a su apreciación frente al desempeño de los Supervisores de Campo, considerando ítems relacionados a la capacidad de solución de problemas, explicación adecuada de la tarea, trato igualitario, respeto, entrega de herramientas, motivación, orden del equipo de trabajo, atención de necesidades fuera del proceso y control de jabas cosechadas. Los resultados de cada encuesta fueron analizados con el Jefe de Producción y las gerencias, desarrollando posteriormente la retroinformación personal a cada Supervisor de Campo. Para Robbins y Judge (2017), la mejora del desempeño grupal debería estar influida por características de un buen líder, por ser quien dirige al grupo al logro de las metas. 


\section{CAPÍTULO III: RESULTADOS DE LA INTERVENCIÓN}

A continuación se describen los resultados cualitativos y cuantitativos alcanzados a partir de las actividades implementadas, las cuales fueron aprobadas por la Gerencia quien además de participar de la programación, fue observador eventual de la implementación, mostrándose interesado por el desarrollo de las actividades.

\subsection{Resultado de las estrategias implementadas para la selección del talento:}

Se incrementó en 94\% la cantidad máxima de candidatos a Operario que participaron en una misma fecha de evaluación, pasando de 90 personas en el año 2017 a 175 en el año 2018, según los registros realizados. Este resultado evidenció que la estrategia de reclutamiento se ajustó a las características particulares de la zona, logrando superar la oferta de los informales e incrementando las probabilidades de encontrar talento. Así también, impactó en los resultados de la empresa, pues se tuvo la posibilidad de contratar a las personas más competentes de la zona para la ejecución de la importante labor de cosecha. Tal como lo señalan Dessler y Varela (2011), la importancia de esta etapa radica en que mientras más candidatos se tenga, más selectivo se podrá ser con la contratación.

Se incrementó el nivel de productividad del personal Operario. Al realizar un seguimiento a la selección, en el año 2017, el Jefe de Producción informó un mayor rendimiento en comparación con los años anteriores, concluyendo que el personal contratado era más productivo. Steiner (como se citó en Mababu, 2014) considera que la productividad actual del grupo es el rendimiento real definido por la productividad potencial, la cual incluye aptitudes, habilidades y conocimientos. Esto demuestra la efectividad de la estrategia de selección que facilitó la contratación de 
personas con competencias alineadas a las necesidades de la operación logrando incrementar la producción de los Operarios. Al tratarse de tareas realizadas en grupos, el resultado del personal nuevo motivó al antiguo a exigirse más. Sin embargo, dentro de las dificultades del proceso, la evaluación con el Test de la Persona bajo la lluvia (Querol y Alcañiz, 2005) tuvo que ser cambiada por el Test de la Figura humana (Portuondo, 1997), debido a que el lugar de origen de los postulantes era la región Sierra del país, donde la lluvia era un factor favorable para ellos. Ambas pruebas proyectivas fueron posteriormente menos valoradas como herramientas de evaluación por tratarse de procesos de selección masivos en los que se perdía información, dificultando su correcta interpretación.

Se redujo el tiempo en el proceso de reclutamiento y selección. Siguiendo con el autor Mazabel (2015), quien considera importante administrar eficientemente los costos y tiempos en un área de selección. La base de datos de candidatos evaluados, alimentada con cada proceso de selección, redujo los tiempos de respuesta para cubrir vacantes en dos situaciones. La primera, ante un requerimiento repentino de personal, pues se contaba con la lista de candidatos que habían pasado por el proceso y no habían sido contratos. La segunda, en el desarrollo de procesos de selección siguientes, pues se contaba con un histórico de resultados de evaluación y verificación de referencias personales de quienes postulaban por segunda vez.

\subsection{Resultado de las estrategias para orientar la formación de los colaboradores}

Se implementaron las capacitaciones necesarias para el negocio, siguiendo el plan anual de capacitaciones. Tal como lo señalan Dessler y Varela (2011), la capacitación debe tener sentido de manera que cada empleado pueda aportar al logro de las metas de la organización. Fue imprescindible entender el negocio para que el 
contenido del plan contribuya con los objetivos del mismo. El orden seguido permitió programar los recursos con el tiempo necesario, coordinar con las jefaturas la disponibilidad de su personal y acceder al histórico de temas por trabajador para identificar de forma rápida la información. El reporte de trabajadores "activos" enviado por el área de Personal ayudó a controlar al personal capacitado, se pudo identificar Operarios que no participaron de la capacitación o que carecían de alguna capacitación necesaria para la ejecución de su función.

El nuevo personal Operario mostró mayor seguridad en su desenvolvimiento, a partir de la inducción. Sabía cómo canalizar sus consultas, tenía menos dudas sobre las condiciones laborales, comprendía con mayor rapidez las instrucciones y mostraba una actitud positiva en campo. Siguiendo a Dessler y Varela (2011), la inducción forma parte de la socialización del trabajador nuevo. El impacto positivo de la capacitación al personal se evidenció en las funciones del Supervisor de Campo, quien pudo orientarse a controlar el cumplimiento de las pautas de cosecha dadas en la Inducción, aclarar dudas del personal en el proceso de cosecha, contar con tiempo disponible para contribuir con el control de la calidad del producto cosechado y corregir errores en el momento. Sin embargo, el proceso de inducción al puesto alcanzó sólo al nivel de Operarios, el personal ingresante a otras áreas era entrenado sobre la marcha por sus jefes directos.

El personal de campo aprendió sobre los temas capacitados. El aprendizaje puede inferirse a partir de la conducta observable y normalmente implica un cambio permanente en el desempeño conductual de la persona, asociado a una experiencia anterior (Gross y Ortiz, 2012). El aprendizaje se evidenció con resultados satisfactorios en las auditorías realizadas por certificadoras internacionales, con respecto a la capacitación del personal ( $\sin$ "no conformidades"), considerando que 
este proceso incluía entrevistas a los trabajadores para validar que habían sido capacitados en los temas requeridos y preguntas de conocimiento para validar que los temas habían sido comprendidos. Así también, los resultados del área de Producción señalaron menos errores de cosecha originados por factores humanos. Finalmente, el resultado de la medición de clima organización del año 2017 mostró un nivel de satisfacción de 3.52 sobre 4.00 (88\%) frente al ítem "puedo aprender cosas nuevas relacionadas con mi trabajo”.

Mejoras en las habilidades de gestión de los Supervisores de Campo. Transcurrido un tiempo de dos meses, se empezaron a evidenciar las mejoras. Los tres Supervisores incrementaron su habilidad para solucionar problemas en $2.34 \%$ (promedio), dos mejoraron en $2.42 \%$ (promedio) su capacidad para explicar de manera adecuada y dos mejoraron en $1.59 \%$ (promedio) el orden de sus equipos de trabajo; las otras mejoras se dieron individualmente en habilidades de motivación $(0.32 \%)$, control $(1.09 \%)$ y trato igualitario (1.65\%). Esto, según la apreciación de los Operarios expresada en las encuestas desarrolladas en mayo y julio del año 2018. Oriza (2012) considera que una visión de desarrollo involucra competencias de liderazgo, lo cual conduce a que la organización sea competitiva y excelente. En este sentido se logró iniciar el desarrollo de las capacidades de liderazgo en los Supervisores de Campo. Sin embargo, entre las dificultades identificadas durante el proceso, la encuesta fue percibida por los Supervisores de Campo como una herramienta de "poder" para los Operarios, pues consideraban que los resultados serían utilizados en su contra; las características educativas de los Operarios generaron la anulación de algunas encuestas y el nivel educativo de los Supervisores de Campo dificultó la comprensión de los resultados. 


\section{CONCLUSIONES}

- Se enfocó la gestión en los procesos relacionados con la producción por ser el área más importante. Se buscó potenciar el rendimiento del personal operario que representa el $80 \%$ de la población y cumple una de las funciones más importantes para el negocio: la cosecha.

- Se pudo conocer el negocio e identificar sus necesidades a partir de la información verbal del personal de campo y de la observación directa de los procesos.

- Se contó con el interés y contribución del Jefe de Producción y Gerencia durante la preparación e implementación de las acciones.

- Se logró incrementar en 94\% la cantidad máxima de candidatos a Operario, participantes de una misma fecha de evaluación, a pesar de la oferta laboral de los "contratistas".

- Se elaboró una base de datos de postulantes y resultados, lo cual redujo tiempos de respuesta para cubrir vacantes no programadas y facilitó la evaluación de los candidatos que se presentaban por segunda vez.

- Se desarrolló un programa de capacitaciones alineado a las necesidades reales del negocio, su implementación permitió mantener la información ordenada y controlar los avances.

- Se incrementó la productividad del personal Operario y se redujo los errores de cosecha causados por factores humanos, según la información del Jefe de Producción.

- Se obtuvo resultados satisfactorios en las auditorías realizadas por certificadoras internacionales, con respecto a la capacitación del personal (sin "no conformidades").

- Se alcanzó una satisfacción de 3.52 sobre 4.00 (88\%) frente al ítem "puedo aprender cosas nuevas relacionadas con mi trabajo" en la medición de clima organizacional del año 2017.

- Se mejoraron las habilidades de gestión de los Supervisores de Campo, principalmente en solución de problemas, explicación adecuada y orden de sus equipos de trabajo, según la apreciación de los Operarios. 


\section{RECOMENDACIONES}

- Comprender la naturaleza del negocio antes de programar o implementar procesos de recursos humanos, a fin de que las acciones se orienten a las necesidades reales del negocio.

- Contar con la Descripción de Puestos antes de dar inicio al reclutamiento a fin facilitar el proceso de Selección. Este documento debe contener las competencias a nivel organizacional y a nivel del puesto descritas en un diccionario de competencias.

- Elaborar los procedimientos de todas las áreas, con el fin de contar con un documento que facilite la adaptación al puesto del nuevo personal y permita replicar el proceso seguido.

- Fomentar la comunicación con el personal de campo, pues además de ser un medio de información para la identificación de necesidades, es un medio de control del alcance de las acciones implementadas y de la satisfacción con las mismas.

- Hacer uso de herramientas poco complejas, en selección y capacitación, que se adapten a las características de todos los participantes y que eviten enfrentarlos a situaciones que los desmotiven a continuar o que provoquen la anulación de su participación.

- Motivar a los responsables de manejo de personal, sean supervisores, jefes o gerentes, a comprometerse con la mejora del clima organizacional y con la promoción de la cultura empresarial que se desea alcanzar.

- Continuar con el trabajo iniciado en el área de Producción y procurar extender su alcance a nivel de todas las áreas de la organización. 


\section{REFERENCIAS}

Banco Mundial. (2017). Tomando impulso en la agricultura peruana. Oportunidades para aumentar la productividad y mejorar la competitividad del sector. Recuperado del sitio de internet del Banco Mundial:

http://documentos.bancomundial.org/curated/es/781561519138355286/Gainingmomentum-in-Peruvian-agriculture-opportunities-to-increase-productivity-andenhance-competitiveness

Chiavenato, I. (2017). Administración de recursos humanos: El capital humano de las organizaciones. México: McGraw-Hill Educación

Dessler, G., y Varela, R. (2011). Administración de recursos humanos: enfoque latinoamericano (5. ${ }^{\mathrm{a}}$ ed.). México: Pearson Educación.

Gross, R. D., y Ortiz, S. M. E. (2012). Psicología: La ciencia de la mente y la conducta. México: El Manual Moderno.

Imbernón, F., Medina, J. L., Arànega, S., Bozu, Z., Jarauta, B. B., y Serrat, A. N. (2016). Diseño, desarrollo y evaluación de los procesos de formación. Madrid: Síntesis.

Mababu, M. R. (2014). Psicología de los grupos. Madrid: Centro de Estudios Financieros Mazabel, C. (2015). Indicadores de recursos humanos y su resultado económico en la empresa: "de lo cualitativo a lo cuantitativo". Lima: Centro de Investigaciones de Recursos Humanos

Ministerio de Agricultura y Riego. Publicaciones y Prensa. (1 de Marzo de 2018). Sector Agricultura seguirá impulsando la economía peruana. Recuperado de http://minagri.gob.pe/portal/publicaciones-y-prensa/noticias-2018/21075-sectoragricultura-seguira-impulsando-la-economia-peruana 
Organización de las Naciones Unidas para la Alimentación y la Agricultura. (2000). Agricultura y diálogo de culturas, nuestro patrimonio común. Recuperado de http://www.fao.org/3/a0015s/a0015s00.htm

Oriza, J. (2012). De jefe a líder: liderazgo basado en la inteligencia emocional y los valores. México: Trillas.

Pimienta, J. (2012). Estrategias de enseñanza - aprendizaje. México: Pearson Educación.

Portuond, J. (1997). La figura humana: test proyectivo de Karen Machover. España: Biblioteca Nueva.

Programa Laboral del Desarrollo. (2015). Informe: Evaluación de la eficiencia de los sistemas de certificación internacional sobre condiciones laborales en empresas agroexportadoras: Los casos de Ica y La Libertad. Lima: Plades.

Querol, S. y Alcañiz, S. (2005). Selección de personal: aplicación del test de la persona bajo la lluvia y análisis grafológico del relato. Buenos Aires: Lugar

Robbins, S. P., y Judge, T. A. (2017). Comportamiento organizacional (17. a. ed.). Ciudad de México: Pearson Educación

Vargas, R. (19 de febrero de 2018). Assessment Center vs. evaluaciones psicológicas: ¿cómo contratar al mejor personal para tu negocio?. Recuperado de https://www.esan.edu.pe/apuntes-empresariales/2018/02/assessment-center-vsevaluaciones-psicologicas-como-contratar-al-mejor-personal-para-tu-negocio/ 


\section{APÉNDICES}




\section{APÉNDICE 1: COMPARACIÓN ENTRE RÉGIMEN LABORAL GENERAL Y RÉGIMEN LABORAL AGRAGRIO}

Comparación entre régimen laboral común y la ley 27360

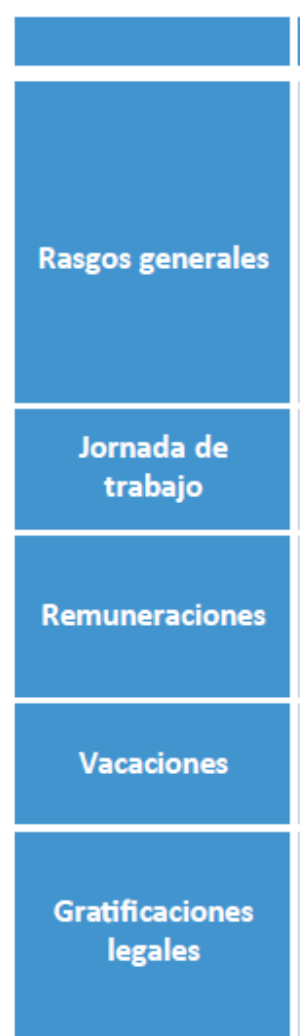

Compensación por Tiempo de Servicios

Indemnización
por despido
arbitrario

Seguro de salud

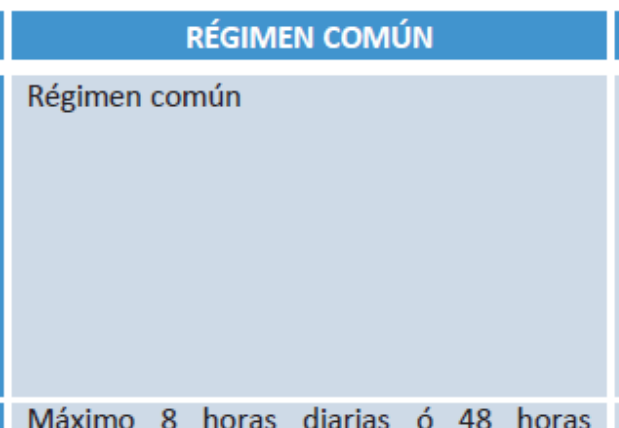

semanales.

Salario no inferior a la Remuneración Mínima Legal. Además, Compensación por Tiempo de Servicios (CTS) y las Gratificaciones

Descanso vacacional remunerado de treinta (30) días calendarios remunerados por cada año de servicios;

Dos gratificaciones al año: una en julio y otra en diciembre, con un monto, en cada caso, equivalente a la remuneración mensual que perciba el trabajador en la oportunidad en que corresponda su pago.

Los empleadores depositan tantos dozavos de la remuneración computable percibida por el trabajador como meses completos haya laborado.

Se pagan 45 días ( $1 \frac{1}{2}$ remuneración) por cada año efectivo de servicios hasta un tope de 360 días.

$9 \%$

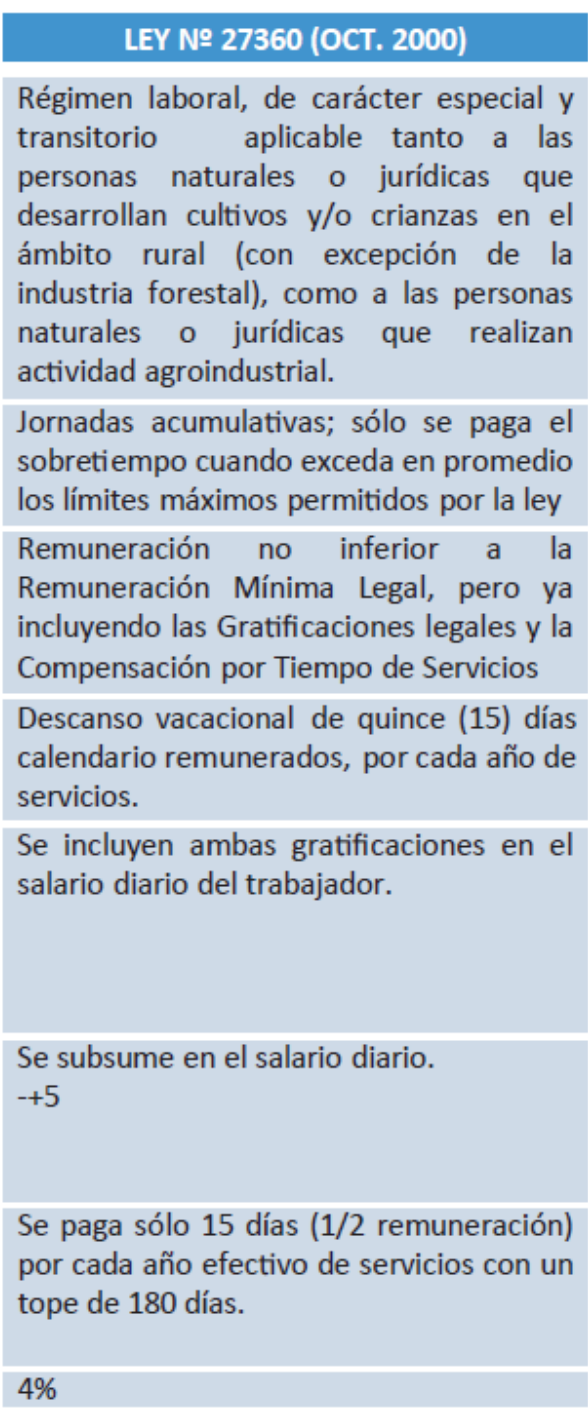

Fuente: Programa laboral de desarrollo, PLADES (2012). 